\title{
Bayesian quantile regression analysis of Korean Jeonse deposit
}

\author{
Eun Jung Nam ${ }^{a}$, Eun Kyung Lee ${ }^{a}$, Man-Suk Oh ${ }^{1, a}$ \\ ${ }^{a}$ Department of Statistics, Ewha Womans University, Korea
}

\begin{abstract}
Jeonse is a unique property rental system in Korea in which a tenant pays a part of the price of a leased property as a fixed amount security deposit and gets back the entire deposit when the tenant moves out at the end of the tenancy. Jeonse deposit is very important in the Korean real estate market since it is directly related to the residential property sales price and it is a key indicator to predict future real estate market trend. Jeonse deposit data shows a skewed and heteroscedastic distribution and the commonly used mean regression model may be inappropriate for the analysis of Jeonse deposit data. In this paper, we apply a Bayesian quantile regression model to analyze Jeonse deposit data, which is non-parametric and does not require any distributional assumptions. Analysis results show that the quantile regression coefficients of most explanatory variables change dramatically for different quantiles. The regression coefficients of some variables have different signs for different quantiles, implying that even the same variable may affect the Jeonse deposit in the opposite direction depending on the amount of deposit.
\end{abstract}

Keywords: real estate, Bayesian analysis, quantile regression, apartment rent

\section{Introduction}

Jeonse is a unique property rental system in Korea in which a tenant pays a part of the price of a leased property as a fixed amount security deposit and gets back the entire deposit when the tenant moves out at the end of the tenancy. Jeonse has developed to meet the mutual interests of the house owners and tenants. Tenants use this system as a step to buy their own houses with guaranteed stable residence and house owners utilize it to finance their assets expecting an increase in property prices (Kim, 2015). Fast industrialization followed by the concentration of population in urban areas has increased the demands for residential properties; however, the availability of Jeonse house has not grown enough to meet the demand. As a result, the shortage of house supplies in cities has continued. It is expected that the rising trend of housing prices are likely to continue (Korea Appraisal Board, 2017).

Stability in the real estate market is important to the nation and the public. A continuous rise in property prices carries inherent risks such as a real estate bubble that may lead to a long-term recession with contagious impacts on the global economy as evidenced by the US subprime mortgage crisis or real estate bubble burst in Japan. Also, hard landing in the real estate market would lead to the worsening of the construction industry which accounts for a large part of the national economy and may cause large losses in the domestic financial industry. Furthermore, household debt would also increase

\footnotetext{
${ }^{1}$ Corresponding author: Department of Statistics, Ewha Womans University, 52, Ewhayeodae-gil, Seodaemun-gu, Seoul 03760, Korea. E-mail: msoh@ewha.ac.kr
}

Published 30 September 2018 / journal homepage: http://csam.or.kr

(c) 2018 The Korean Statistical Society, and Korean International Statistical Society. All rights reserved. 


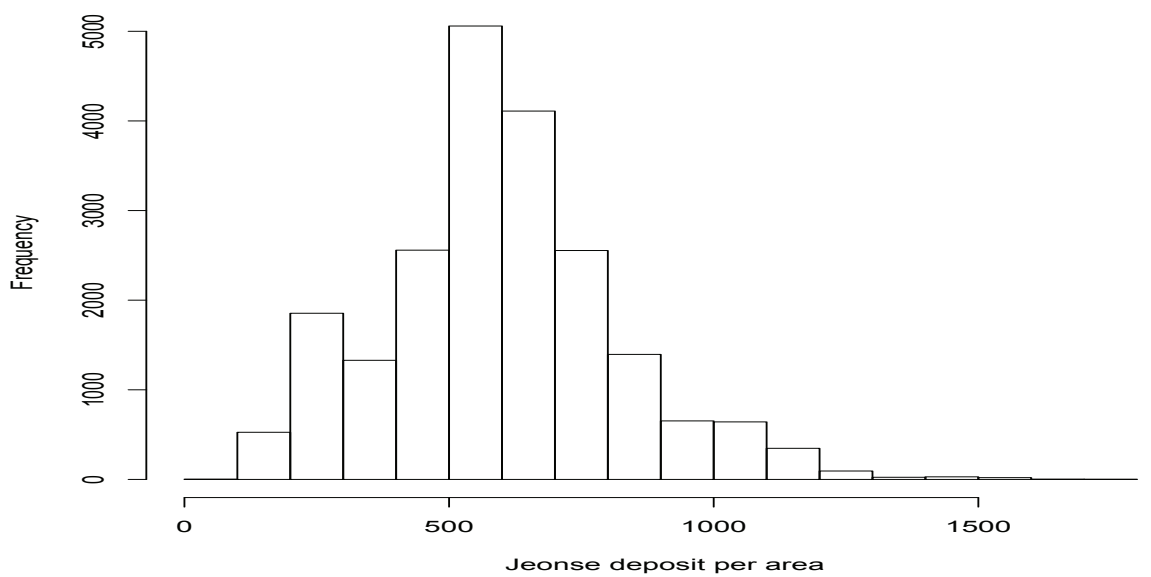

Figure 1: Histogram of Jeonse deposit per area.

while curbing the consumption, creating a vicious cycle of economic deterioration. Considering the critical role of the real estate market to the national economy, it is important to develop useful real estate market information systems that include information on Jeonse deposit and monthly apartment rents (Han, 2016).

Research has been conducted on the Korean real estate market. In particular, price changes for apartments located in Seoul have frequently been study subjects since the Seoul apartment prices are the leading indicator of housing prices nationwide. Most research on apartment prices used a hedonic price model to understand the sales price and the Jeonse deposit of apartments in Seoul (Kim and Lee, 2013; Park and Rhim, 2010) and utilized four types of information (transaction, apartment property, infrastructure, and macro-economic status) as factors that affect housing price (Kim et al., 2000).

Most current studies on the real estate market focus on the conditional mean of the apartment prices given the factors that affect the prices, using the mean regression model. The mean regression model is valid under strong assumptions of normality and homoscedasticity. However, Jeonse deposit data shows obvious skewness and heteroscedasticity that makes the mean regression model inappropriate for the analysis of Jeonse data (Figure 1).

In this paper, we apply a Bayesian quantile regression model to analyze apartment Jeonse deposit data in Seoul. Using the quantile regression model, we study the effect of explanatory variables on a given quantile of the response variable. By changing the quantile, we can investigate the whole distribution, not just the mean, of the response variable (Koenker and Basset, 1978). Also, the quantile regression does not require normality nor homoscedasticity assumptions. We employ a Bayesian approach to fit quantile regression model to Jeonse data using Markov chain Monte Carlo (MCMC). The main advantage of the Bayesian quantile regression is that it is convenient to find the posterior distribution via MCMC algorithms and useful prior information may be utilized (Koenker and Machado, 2001).

Unlike most existing studies on the Korean real estate market data that consider only static factors such as apartment properties and infrastructures (Kim, 2014), we include temporal factors such as the year and the quarter of each year that a transaction occurred, the average amount of Jeonse deposit and the volume of transactions of the previous time point. Inclusion of these time-related variables would help in better understanding Jeonse deposit data, which is clearly time series data.

The rest of this paper is organized as follows. Section 2 presents a brief description of Bayesian 
quantile regression analysis. Section 3 describes the data and variables used in the study. Section 4 presents analysis results. Section 5 gives the conclusion.

\section{Bayesian quantile regression}

The commonly used mean regression model assumes the following relationship between the response variable $y_{i}$ and a $k$-dimensional vector of explanatory variables $x_{i}$ :

$$
\begin{aligned}
& y_{i}=x_{i}^{\prime} \beta+\epsilon_{i}, \\
& \epsilon_{i} \sim N\left(0, \sigma^{2}\right) .
\end{aligned}
$$

It means that $y_{i}$ follows a normal distribution with mean $E\left(y_{i} \mid x_{i}\right)=x_{i}^{\prime} \beta$ and a constant variance regardless of the $x_{i}$ value. Here $\beta$ is a $k$-dimensional vector of regression coefficients.

Let $Q_{p}\left(y_{i} \mid x_{i}\right)$ be the $p^{\text {th }}$ quantile of $y_{i}$ given $x_{i}$. The quantile regression model assumes

$$
Q_{p}\left(y_{i} \mid x_{i}\right)=x_{i}^{\prime} \beta_{p}
$$

where $\beta_{p}$ is a $k$-dimensional vector of regression coefficient which depends on $p$. If the assumption of the mean regression model (the normality and the homoscedasticity) holds, $Q_{p}\left(y_{i} \mid x_{i}\right)$ is equal to a constant plus $x_{i}^{\prime} \beta$. The effect of an explanatory variable on the $p^{\text {th }}$ quantile of $y_{i}$ is the same for any $p$. However, either the normality or the homoscedasticity assumption does not hold, the effect of an explanatory variable may change for different $p$ 's and hence it may not be appropriate to assume the same regression coefficient for different $p$ 's.

Using the quantile regression model, the overall distribution of the response variable rather than just the mean can be investigated by changing $p$ 's. In addition, the quantile regression model does not assume the distribution of $y_{i}$ and is therefore robust to outliers and/or non-normal distribution of $y_{i}$.

Consistent estimator of $\beta_{p}$ can be obtained by minimizing the loss function

$$
\sum_{i=1}^{n} \rho_{p}\left(y_{i}-x_{i}^{\prime} \beta_{p}\right)
$$

where $\rho_{p}(\cdot)$ is the check loss function given by $\rho_{p}(u)=u(p-I(u<0)), I()$ is the indicator function.

Bayesian quantile regression model assumes

$$
y_{i}=x_{i}^{\prime} \beta_{p}+\varepsilon_{i}, \quad \varepsilon_{i} \sim \operatorname{ALD}(p),
$$

where $\operatorname{ALD}(p)$ denotes the asymmetric Laplace distribution (ALD) with $p^{\text {th }}$ quantile at 0 , having the probability density function $f(\varepsilon)=p(1-p) e^{-\rho_{p}(\epsilon)}$ (Koenker and Machado, 2001). From model (2.3), the likelihood function of $\beta_{p}$ is given as follows.

$$
l\left(\beta_{p} \mid x, y\right)=\prod_{i=1}^{n} f_{p}\left(y_{i} \mid \beta, x_{i}\right)=p^{n}(1-p)^{n} e^{-\sum_{i} \rho_{p}\left(y_{i}-x_{i}^{\prime} \beta_{p}\right)}
$$

Note that maximizing the likelihood (2.4) is equivalent to minimizing (2.2).

For Bayesian inference, the prior distribution of $\beta_{p}$ may be given as $\beta_{p} \sim N\left(\beta_{0 p}, \sum_{0 p}\right)$. Combining the likelihood and the prior, the posterior distribution of $\beta_{p}$ can be derived. However, it can be easily seen that analytic derivation of the posterior density function of $\beta_{p}$ is not easy. To get around this difficulty, we apply MCMC to generate posterior samples of $\beta_{p}$ and Bayesian inference can be done based on MCMC samples. See Park and Oh (2016), Oh et al. (2016a, 2016b) for details of MCMC algorithms for Bayesian quantile regression analysis. In this study we use Just Another Gibbs Sampler (JAGS, http://mcmc-jags.sourceforge.net/) package to generate MCMC samples. 
Table 1: Resource of data

\begin{tabular}{lll}
\hline \hline \multicolumn{1}{c}{ Data } & \multicolumn{1}{c}{ Web site } & \multicolumn{1}{c}{ URL } \\
\hline Apartment property & Apartment Price Management System of the MOLIT & http://www.k-apt.go.kr/ \\
Transaction & Real Transaction Price Information System of the MOLIT & http://rt.molit.go.kr/ \\
Infrastructure & Seoul Open Data Plaza & http://data.seoul.go.kr/ \\
Macroeconomic status & Economic Statistic System of Bank of Korea & http://ecos.bo.or.kr/ \\
\hline
\end{tabular}

Table 2: Data description

\begin{tabular}{cll}
\hline \hline Category & Variable & \\
\hline Response & price_area & Jeonse price per area \\
\hline Apartment & area & Exclusive area \\
property & floor & floor \\
& apart_age & Year of construction of apartment \\
& CCTV_n & Number of CCTVs per household \\
& parking_n & Number of parking spaces per household \\
& metro & Time to the nearest subway station \\
\hline Transaction & year & Year of transaction \\
& quarter & Quarter of transaction \\
& last_n & Number of apartment units of similar floor area (in pyeong) sold/bought in the \\
& last_price & previous month \\
& Average sales price of apartment units of similar floor area (in pyeong) \\
& sold/bought in the previous month \\
\hline Infrastructure & park & Whether a department store is located in the vicinity of subject apartment \\
& pop_den & Whether a park is located in the vicinity of subject apartment \\
& CPI & Consumer price index of the previous month \\
& market_rate & Market interest rate of the previous month \\
\hline Macroeconomic & &
\end{tabular}

\section{Data description}

\subsection{Data collection}

We focus on the apartments located in Gangnam-gu, Seoul, which is known to be the leading nationwide apartment indicator for Jeonse deposits and sale prices (Kim and Lee, 2013). We use data collected from 2014 to 2016. Previous study of Choi (2017) showed that four major categories which affect house prices are transaction, apartment property, infrastructure and macroeconomic status. We consider these four categories in our study that are obtained from sources given in Table 1. In the table, MOLIT represents the Ministry of Land, Infrastructure and Transportation of Korea.

In this study, a total of 21,547 observations is obtained. There are 345 observations with missing values, which account for a very small fraction (about 1.60\%) of the total data. After excluding those missing observations, a total of 21,202 observations is used for the analysis. Every explanatory variable is standardized to have a mean 0 and variance 1 before analysis for ease of comparison.

\subsection{Description of variables}

In each of the four categories, there exist many variables. Table 2 gives the variables finally used in the analysis. We give detailed description of the variables in the rest of this subsection.

- Jeonse deposit per area: Jeonse deposit tends to be proportional to the area of the leased apartment. Therefore, this study uses Jeonse deposit per area $\left(100,000 \mathrm{won} / \mathrm{m}^{2}\right)$ as the response variable. 
- Area: One of the significant determinants that affects the price of real estate is the area.The deposit divided by area is used as the response variable in this study. However, the increase level in deposit of larger apartments may not be the same as that of smaller apartments, meaning that deposit per area may not be independent of the area. Thus, the area is added as an explanatory variable.

- Floor: Floor is a key factor in determining the amount of sunlight and a view of the residence. In the past, middle floors were preferred and were traded at the higher price. However, increasing number of tall story buildings and the advancement of heating systems have changed the preference and people now seem to prefer higher floors. Thus, floor is included as an explanatory variable.

- Age of apartment: In general, the apartments which were built more recently are more expensive. Apartment age has a big impact on management expenses. In addition, recently-built apartments tend to have more convenient and the latest facilities such as elevator reservation systems and fitness centers, which attract people. We use the apartment age as an explanatory variable.

- Number of CCTVs per household: Security is a critical factor for resident satisfaction (Shim et al., 2014). Security appears to have impact on the decision of residential area and be directly linked to the sales price. Therefore, this study includes the number of CCTV per household.

- Number of parking spaces per household: It was found that parking space has a significant impact on apartment price, but the level of impact was different by each quantile of sales price (Kim, 2014). So it would be reasonable to assume that Jeonse deposit is also influenced by parking space. The number of parking units per household is considered as an explanatory variable.

- Distance to public transportation: In general, the price of apartment differs according to the level of accessibility to public transportation. However, residents in expensive high-end apartments usually use their own cars as transportation means. Thus, the distance to public transportation may have different impact on different quantiles of the Jeonse deposit per area. To check this, we include "time to nearest subway station" as an explanatory variable.

- Time of transaction (Year and Quarter): The year, the month and the day of a transaction are available from the MOLIT. A simple analysis on the relationship between the day of transaction and the deposit per area shows that the day is not a significant factor on deposit per area. However, Jeonse deposits tend to increase by year with a seasonal effect (Figure 2). Thus, we include the year and the quarter in each year as explanatory variables.

- Transaction of apartments in similar conditions during the previous month (Number of transactions and the average deposit): The amount of deposits per area is a time series data which changes over time, and the previous Jeonse deposit transactions would impact current and future Jeonse transactions. Thus, we use transaction data on apartments in similar conditions during the previous month as an explanatory variable. Tenants tend to consider the location and the environment of the apartment as primary factors. Thus, the number of transactions and the Jeonse deposit per area of neighboring apartments in the previous month are considered as explanatory variables.

- Convenience (Existence of department store, park, and child care facilities): The vicinity area may also influence apartment Jeonse deposit as an environmental factor. Especially, the presence or absence of large shopping malls (including department stores) indicates the convenience level of living and appears to have a significant influence on Jeonse deposit. The distance to a park also has a significant effect (Choi, 2017). Households with infants are actively involved in the Jeonse 


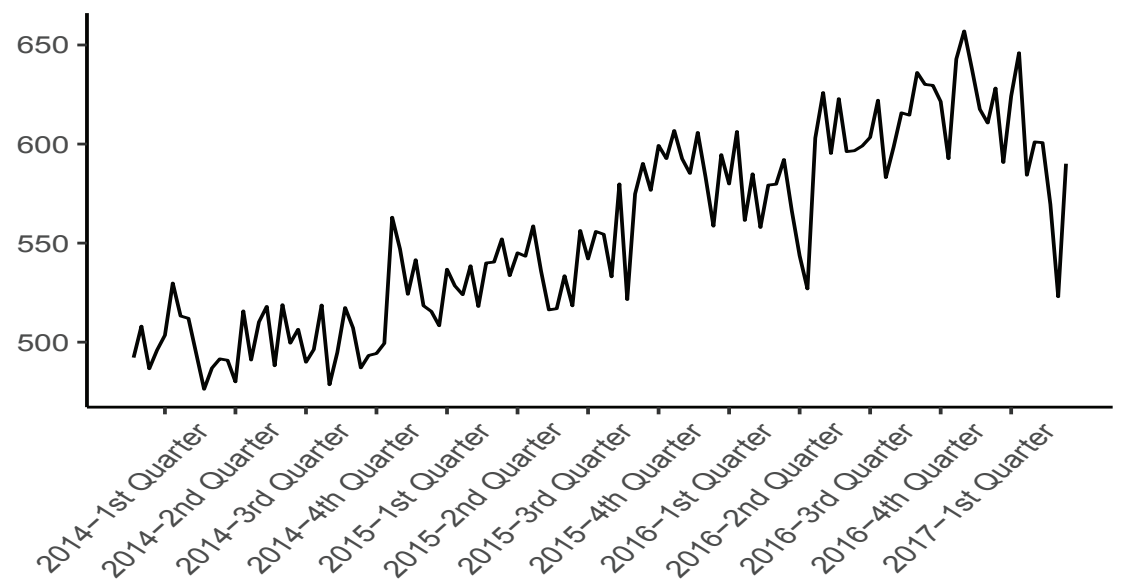

Figure 2: Time series plot of the mean Jeonse deposit per area.

market, hence child care facilities may have influence on Jeonse deposits. This study includes these variables as explanatory discrete variables: 1 is assigned if the facility exists in the vicinity and 0 if not.

- Regional environment (Average population density): Previous studies suggest that the regional environment has a significant effect on the real estate market (Yang, 2014). It is presumed that people are likely concentrate in quality residential areas (where high-end apartments are located). The average population density by county (Dong) is added as a variable tocheck this assumption.

- Macroeconomic status (Consumer price index and market interest rate): The real estate market interacts with economic conditions such as interest rates, consumer price index (CPI), and stock price index prevailing at time of transaction (Song et al., 2012). In particular, apartment price in Seoul is strongly related to the KOSPI and has a strong negative correlation with interest rates (Choi et al., 2014). The economic conditions may also affect Jeonse deposit and may show different impact for different quantiles. In this study, we consider CPI and the market interest rate of the previous month as explanatory variables.

- Other variables: Other variables not mentioned above are heating system, corridor type, and management method of the apartment complex. These variables are also known to have significant impacts on apartment price; however, the analysis of variance with the area as the response variable shows that these variables are strongly correlated with the area. Therefore, we exclude these variables from the analysis to avoid multicollinearity.

\section{Analysis results}

\subsection{Overview}

We conducted a Bayesian quantile regression analysis for $p=0.05,0.10,0.15, \ldots, 0.95$. We ran MCMC using JAGS package with 3 MCMC chains and from each chain we obtained 30,000 samples after 10,000 burn-in with no thinning. Traceplots and Gelman statistics (all lower than 1.1) showed that the algorithm seem to have appropriately converged. Figure 3 presents traceplots of the MCMC 

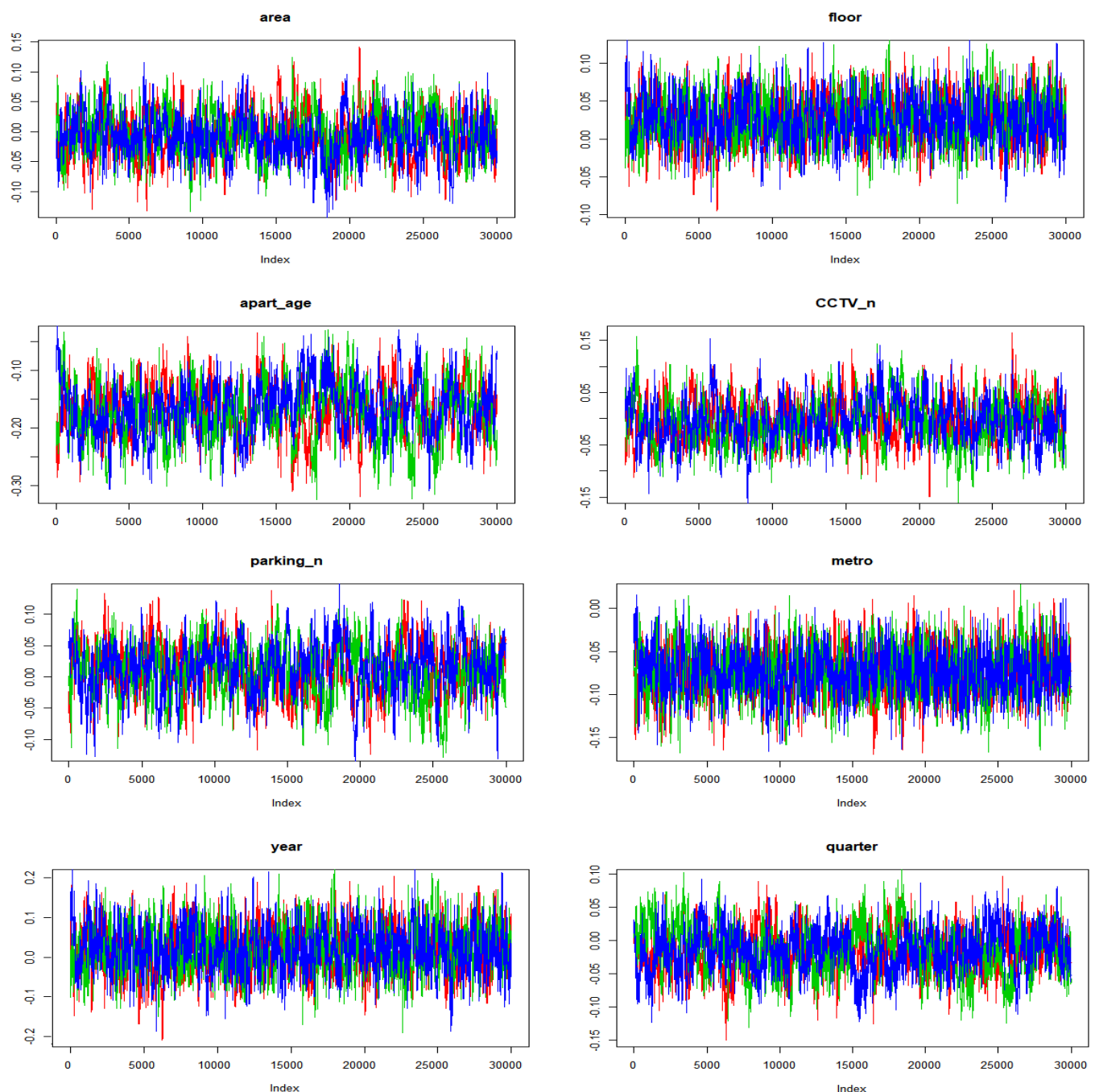

Figure 3: Traceplots of the Markov chain Monte Carlo samples of the coefficients for 8 variables when $p=0.5$.

samples of the coefficients for 8 variables when $p=0.5$. Trace plots for other coefficients show similar patterns and are omitted for space.

For the priors of $\beta_{p} \sim N\left(\beta_{0 p}, \Sigma_{0 p}\right)$, we fitted a linear regression model to the data and use the least squares estimates $\hat{\beta}$ for $\beta_{0 p}$ and 100 times the variance of $\hat{\beta}$ for $\Sigma_{0 p}$.

In Figure 4, the solid line represents the Bayesian quantile regression coefficient estimates and the gray area represents an approximate $95 \%$ highest posterior interval (HPD) for each variable. Table 3 presents estimates of the regression coefficients for quantiles $0.1,0.2, \ldots, 0.9$. The figure shows the dramatic changes of the regression coefficients for different quantiles. The great variability of the regression coefficients imply that the same variable has different (even in the opposite direction for some variables) effects in Jeonse deposit depending on the quantiles.

We describe the impact of explanatory variables for each category. 

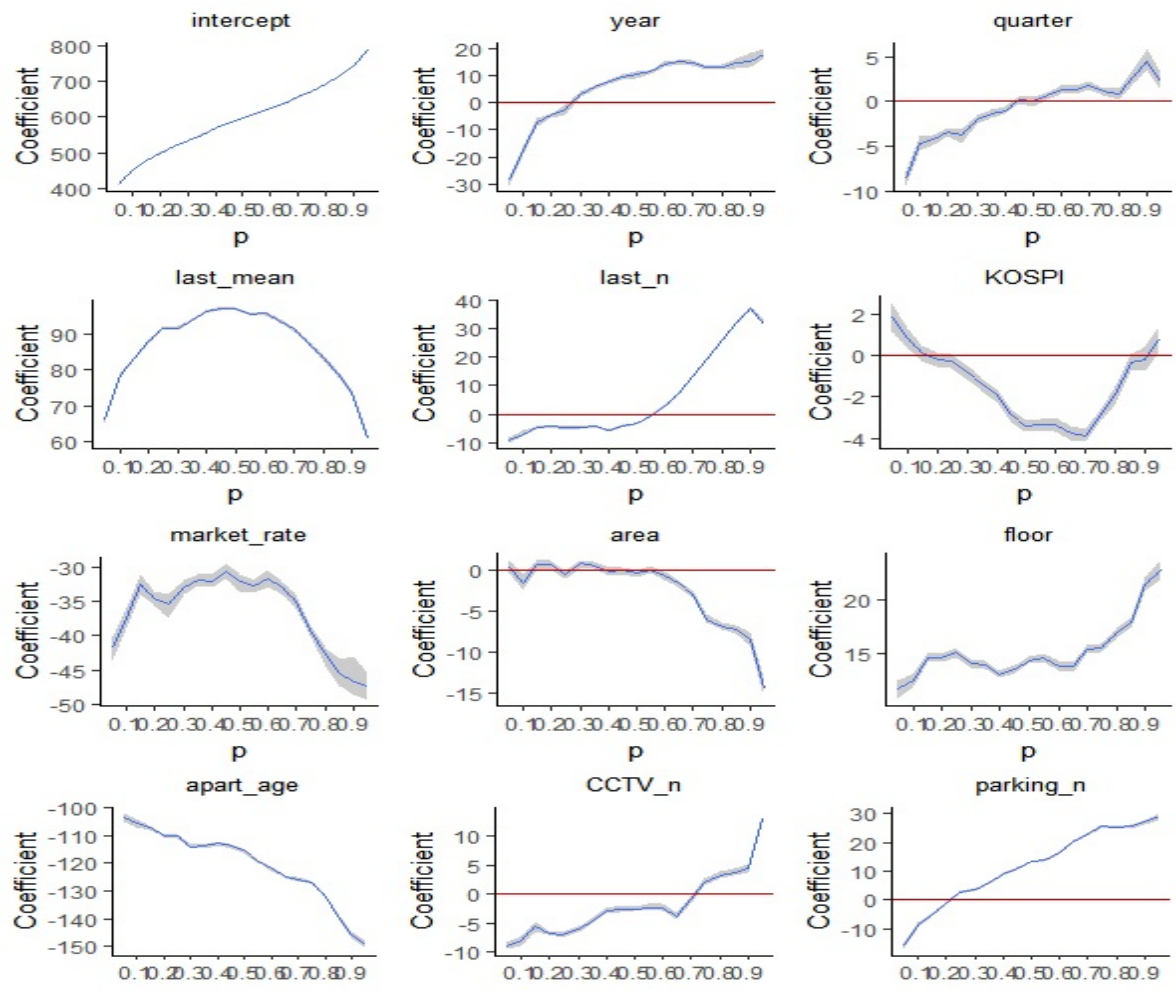

$\mathrm{p}$
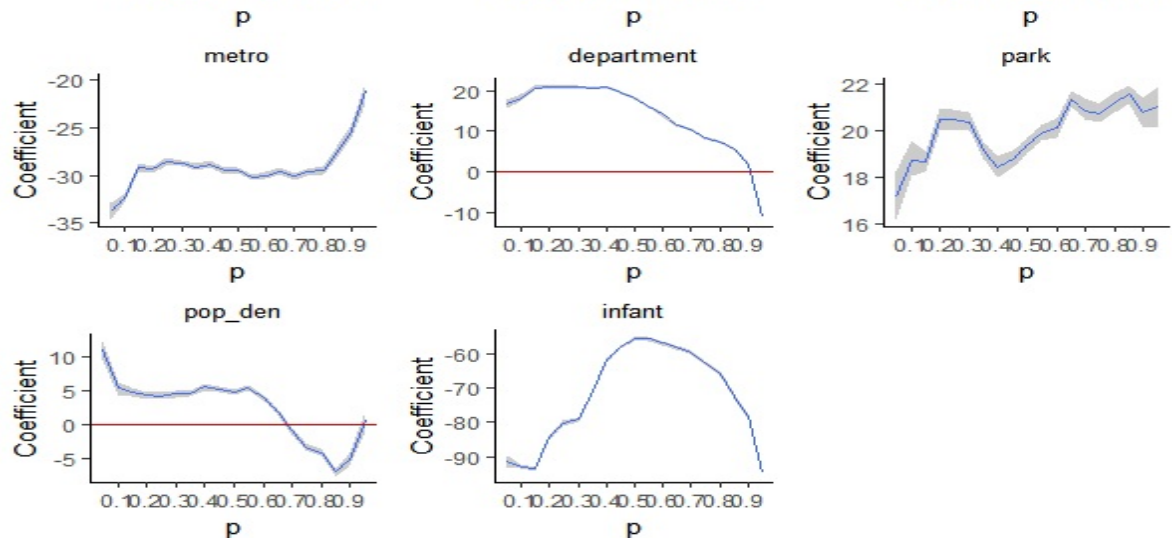

p

Figure 4: Regression coefficients of variables by quantiles.

\subsection{Apartment property}

The area has no effect for the quantiles below 0.6 and has negative effects for the quantiles higher than 0.6. The negative effect accelerates as p gets higher. The effect of area on Jeonse deposit is encountered fully in the deposit per area for low quantiles. However, the deposit per area decreases as the area increases for a high deposit per area apartment.

The coefficient of the floor is always positive and bigger in the higher quantiles. It can be under- 
Table 3: Estimates of the regression coefficient

\begin{tabular}{lrrrrrrrrr}
\hline \hline \multicolumn{1}{c}{$p$} & \multicolumn{1}{c}{0.1} & \multicolumn{1}{c}{0.2} & \multicolumn{1}{c}{0.3} & \multicolumn{1}{c}{0.4} & \multicolumn{1}{c}{0.5} & 0.6 & 0.7 & 0.8 & 0.9 \\
\hline year & -17.87 & -4.77 & 3.26 & 7.81 & 10.53 & 14.28 & 14.78 & 13.43 & 15.06 \\
quarter & -4.69 & -3.40 & -1.97 & -1.04 & 0.12 & 1.32 & 1.82 & 0.90 & 4.39 \\
last_mean & 78.74 & 87.94 & 91.81 & 96.27 & 97.13 & 95.82 & 91.58 & 83.33 & 73.97 \\
last_n & -6.98 & -4.11 & -4.79 & -5.61 & -3.45 & 2.53 & 13.03 & 26.25 & 37.31 \\
KOSPI & 0.81 & -0.18 & -0.84 & -1.93 & -3.41 & -3.34 & -3.85 & -1.92 & -0.19 \\
market_rate & -37.56 & -34.56 & -32.88 & -32.04 & -32.12 & -31.77 & -35.02 & -42.82 & -46.63 \\
area & -1.53 & 0.74 & 0.79 & -0.12 & -0.38 & -0.62 & -2.84 & -6.84 & -8.46 \\
floor & 12.53 & 14.61 & 14.08 & 13.07 & 14.38 & 13.87 & 15.4 & 16.9 & 21.45 \\
apart_age & -105.26 & -110.01 & -113.99 & -112.76 & -115.32 & -122.02 & -125.6 & -131.89 & -145.60 \\
CCTV_n & -8.01 & -6.65 & -5.99 & -2.75 & -2.52 & -2.31 & -1.02 & 3.24 & 4.33 \\
parking_n & -8.51 & -1.11 & 3.75 & 9.09 & 13.25 & 16.21 & 22.62 & 25.15 & 26.89 \\
metro & -32.4 & -29.36 & -28.74 & -28.81 & -29.55 & -30.02 & -30.13 & -29.49 & -25.35 \\
department & 18.3 & 20.77 & 20.96 & 20.95 & 18.02 & 14.13 & 10.41 & 7.21 & 1.84 \\
park & 18.78 & 20.52 & 20.41 & 18.47 & 19.4 & 20.16 & 20.91 & 21.27 & 20.80 \\
pop_den & 5.30 & 4.24 & 4.47 & 5.48 & 4.71 & 3.91 & -0.97 & -4.19 & -5.27 \\
infant & -92.83 & -84.44 & -79.18 & -62.01 & -55.82 & -56.94 & -59.68 & -65.88 & -78.16 \\
\hline \hline
\end{tabular}

stood that preference for a high floor affect Jeonse deposit more in expensive apartments.

The coefficient of the apartment age is negative and becomes more pronounced in expensive apartments. It appears that the deposit tends to decrease for older apartments and become more expensive for newer apartments.

The number of CCTVs per household and the number of parking lots per household show a similar pattern. The coefficients of the two variables increase, changing from negative to positive.

The time need to reach a subway station, which represents the distance from public transportation, has negative coefficients in all quantiles and seems to have almost the same coefficient except for quantiles over 0.8. The coefficients are close to zero for extremely large quantiles and the impact of public transportation on the deposit is weak. It may be because household income tends to be high in high-priced apartments where residents may have several cars and do not depend on the convenience of public transportation.

\subsection{Transaction}

The transaction year and the transaction quarter have similar effects on the response variable. The coefficients of both variables have negative values in low quantiles that gradually increase and have large positive values in high quantiles. This implies that deposit decreases for cheap apartments but increases for expensive apartments over time. This bipolar trend accelerates as the quantile $p$ approaches the extremes.

The previous month's deposit per area has positive effects on the deposit of the current month in all quantiles and the positive effect is particularly strong in the middle quantiles. This means that the higher the deposit in the previous month, the higher the deposit in the current month and this phenomenon is pronounced in the middle quantiles

The previous month's transaction volume (in numbers) has strong positive effects on Jeonse deposit in high quantiles, while it has a moderate negative effect in lower quantiles.

\subsection{Infrastructure}

The existence of parks around apartments has a positive effect, and the existence of department stores also has a positive effect except for $p$ larger than 0.9. The magnitude of the positive effect is reduced 
as the quantile increases for department stores.

The deposit tends to increase with the increase of population density for quantiles below 0.7 . This tendency is stronger for low priced apartments. It is presumed that high deposit means a favorable residential environment for tenants and that the population tends to be concentrated in such environment. However, the deposit decreases with the increase of population density in a high priced apartment. This may imply that a quiet residential environment has higher value in a high-priced apartment.

The coefficient of using infant care centers are always negative, and the negative impacts are relatively smaller at the middle quantile.

\subsection{Macroeconomic status}

The effect of KOSPI decreases for $p$ between 0 and 0.7 (from positive to negative) and then increase for $p$ between 0.7 and 0.9 (from negative to positive). Except for quantiles at both ends, Jeonse deposit tends to decrease as stock prices increase. This may be because tenants wish to lower the deposit and use the money to engage in investment activities that include equity investments.

The market interest rate has a negative effect across all quantiles but the level of impact gets strong for quantiles greater than 0.8 . The negative relationship between deposit and market interest rate is clear on low and high ends of quantiles.

\section{Conclusion}

Research on the real estate market in Korea have been actively performed since the price of real estate is highly related to national policy and the economy. In this study, we have conducted a Bayesian quantile regression analysis on the Jeonse deposit of apartments located in Gangnam-gu which are presumed to lead the domestic apartment market. We have studied the effect of each explanatory variable on Jeonse deposits for various quantiles.

The study reveals that the average Jeonse deposit of a similar apartment of the previous month, the floor level and the presence of parks always has a positive impact on the deposit. On the other hand, market interest rates, apartment age, time to the nearest subway station and use of infant care facilities have a negative impact. Other factors such as the time of transaction, the number of transactions of the previous month, the KOSPI index, the area, the number of CCTVs, the number of parking spaces per household and the population density have effects in the opposite direction, depending on the quantiles.

Model (2.1) assumes a linear relationship between the quantiles of $Y$ and each explanatory variable $x_{j}, j=1, \ldots, k$. To check the linearity for the given data, we divided the range of each $x_{j}$ into $5-7$ intervals of equal lengths, computed $(0.25,0.5,0.75)$ quantiles of y $\left(Q_{p}\right)$ in each interval, and plotted $Q_{p}$ vs $x_{j}$. The plot shows approximate linear trends especially in the central parts of $x_{j}$ where most data are concentrated. Thus, the linear quantile regression model (2.1) seems to be reasonable for this data. Note, however, that if the linearity is seriously violated even after appropriate transformations, one may often consider non-parametric quantile regression models to account for non-linear relationships.

\section{Acknowledgements}

This research was supported by Basic Science Research Program through the National Research Foundation of Korea (NRF) funded by the Ministry of Education, Science and Technology (No. NRF2016R1A2B4008914). 


\section{References}

Choi C (2017). A study of the determinants of Seoul apartments sale price : focus on sale price the top five districts in Seoul city (Master thesis), Kun Kook University, Seoul.

Choi JI and Lee OD (2014). Correlation analysis among the price of apartments in Seoul, stock market and main Economic Indicators \& industry group indices on the stock market, Journal of Digital Convergence, 12, 45-59.

Han DH (2016). A Study on Problems of Lump-sum Deposit-based Lease System in Housing market of Korea and Improvement Measures (Master thesis), Gwangju University, Gwangju.

Kim JH (2014). The valuation effects of housing attributes in Korea: a quantile regression analysis, Journal of Korean Industrial Economic Association, 27, 173-195.

Kim JY (2015). Comparative analysis between Chonsei Korea and Anticretico in Bolivia, Research for Human Settlements, 85, 41-53, Korea Research Institute for Human Settlements.

Kim JI and Lee SW (2013). Does the Seoul apartment rent price lead the Jeongkuk apartment rent price? Journal of CEO and Management Studies, 16, 1-22.

Kim TY, Jang CH, and Yoon JH (2000). Determinants of apartment price: the case study of Taegu city, Environmental Research, 19, 27-36, Environmental Research Institute, Yeungnam University, Kyungsan, Korea.

Koenker R and Basset G (1978). Regression quantile, Econometrica, 46,33-50.

Koenker R and Machado JAF (2001). Goodness of fit and related inference processes for quantile regression, Journal of the American Statistical Association, 94, 1296-1310.

Korea Appraisal Board (2017). 2017 Korea real estate market prediction report, Korea Appraisal Board, 2017.07.14.

Oh MS, Choi J, and Park ES (2016a). Bayesian variable selection in quantile regression using the savage-dickey density ratio, Journal of the Korean Statistical Society, 45, 466-476.

Oh MS, Park ES, and So BS (2016b). Bayesian variable selection in Binary quantile regression, Statistics \& Probability Letters, 118, 177-181.

Park ES and Oh MS (2016). Bayesian quantile multivariate receptor modelling, Chemometrics and Intelligent Laboratory Systems, 159, 174-180.

Park WS and Rhim BJ (2010). A study on the factors affection apartment price by using Hedonic price model, Journal of Korea Real Estate Society, 28, 245-272.

Shim JY, Lim BH, Lee SY, and Ji NS (2014). A comparative study on the housing satisfactions and influence factors between residents of apartment and single housing, Geographical Journal of Korea, 48, 1-16

Song K, Jung M, and Lee S (2012). A study on the relationship between housing purchase price composite index and Korea stock price index \& industry group indices on the stock market, Journal of Korea Real Estate Society, 48, 77-93.

Yang SC (2014). A study on the single-family house price determinants analyzed by quantile regression: in case of locating single family houses in Seoul, Journal of Korean Geographical Society, 49, 690-704 